\title{
UN ESTUDIO COMPARATIVO SOBRE EL PENSAMIENTO PROFESIONAL Y LA «ACCIÓN DOCENTE» DE LOS PROFESORES DE CIENCIAS DE EDUCACIÓN SECUNDARIA. PARTE II
}

\author{
MARTÍNEZ AZNAR, M.M. ${ }^{1}$, MARTÍN DEL POZO, R. ${ }^{1}$, RODRIGO VEGA, M. ${ }^{1}$, \\ VARELA NIETO, M.P. ${ }^{2}$, FERNÁNDEZ LOZANO, M.P. ${ }^{2}$ y GUERRERO SERÓN, A. ${ }^{3}$ \\ ${ }^{1}$ Departamento de Didáctica de las Ciencias Experimentales \\ ${ }^{2}$ Departamento de Psicología Evolutiva y de la Educación \\ ${ }^{3}$ Departamento de Sociología VI \\ Facultad de Educación. Universidad Complutense de Madrid. Rector Royo Villanova, s/n. \\ 28040 Madrid
}

\begin{abstract}
Resumen. Este artículo es la segunda parte de una investigación más amplia sobre el pensamiento del profesor (Martínez et al., 2001). En este caso el estudio gira en torno al análisis de las diferencias y similitudes entre dos muestras de profesores de ciencias de educación secundaria que difieren en sus años de docencia y en su trayectoria profesional, definida por la realización de cursos de formación permanente de alta cualificación científica y didáctica. En un primer apartado se analizan facetas relacionadas con la motivación y la satisfacción en la realización de su tarea, la manera de entender la formación del profesor y sus percepciones sobre diferentes factores profesionales. A continuación se aborda lo que «dicen hacer» en relación a aspectos curriculares referidos a contenidos, metodología y evaluación. A partir del análisis de las respuestas a los cuestionarios elaborados se plantean una serie de reflexiones que buscarían mejorar el «desarrollo profesional» de este colectivo de profesores.

Palabras clave. Pensamiento del profesor, formación de profesores de ciencias de secundaria, concepciones del profesorado.
\end{abstract}

Summary. This article is a part of a wider research on secondary science teachers' beliefs. Through the use of a questionnaire data were obtained from 103 secondary science teachers in service with different degrees of experience. Analysis of data are discussed in terms of their implication in teacher training in service.

Keywords. Teacher thinking, secondary science teacher education, teachers' conceptions.

\section{INTRODUCCIÓN}

El presente trabajo es complementario del ya publicado en esta revista (Martínez et al., 2001)y ha sido elaborado a partir de una investigación financiada por el $\mathrm{CIDE}^{1}$ cuya finalidad fundamental era contribuir a clarificar, en la medida de lo posible, el diseño de la formación inicial del profesor de ciencias de educación secundaria y a revisar la formación permanente de ese grupo profesional (Martínez et al., 1997). En el artículo citado se elaboró una amplia revisión del marco teórico que sustenta las investigaciones sobre el pensamiento del profesor. Se destacaron estudios relativos al conocimiento científico del profesorado, sus concepciones sobre el aprendizaje, la enseñanza y los aspectos curriculares propios de su desarrollo profesional. También se contempló la gradación en la construcción del conocimiento profesional desde perspectivas más simplificadoras concretadas en los modelos didácticos tradicionales, hasta planteamientos más elaborados que en general podrían servir como hipótesis orientadora de los procesos de formación del profesorado.

En el trabajo anterior se presentaron los resultados más relevantes de un estudio realizado con futuros profesores (estudiantes del CAP) y en el presente aparecen los relativos a las similitudes y las diferencias en relación con las percepciones profesionales y a lo que dicen hacer 
-que nosotros denominaremos acción docente-grupos de profesores de educación secundaria que trabajan en los departamentos de ciencias y que difieren en los años de ejercicio docente y en el hecho de tener una trayectoria profesional ligada a experiencias de formación permanente de alta cualificación.

En España como en otros países de nuestro entorno, se han hecho en los últimos diez años grandes esfuerzos para adaptar la formación permanente a las nuevas tendencias didácticas (Guerrero y Feito, 1996). En línea con todo ello, dentro de las propuestas institucionales que han surgido y, en concreto en el campo de la didáctica de las ciencias, nos parecen de sumo interés los llamados Cursos de Actualización Científica y Didáctica (ACD) de Nivel $A$, entre otras razones porque han supuesto un intento serio de integración de la teoría y la práctica profesional. Además, como rasgo peculiar de estos cursos, por su interés para nuestra investigación, debemos resaltar que a ellos accedieron profesores seleccionados por su experiencia docente consolidada y por sus intereses manifiestos en la formación permanente y en la reforma educativa. Recordemos también que estos cursos de alta cualificación tienen su máximo esplendor a partir de 1993, al amparo de una Orden del MEC del 26/ 4/1993 por la que se convocan cursos de actualización científica y didáctica. Su diseño es de tres fases, que duran un curso escolar (150 horas): las dos primeras de tres y dos semanas, en julio y septiembre respectivamente, tienen un carácter marcadamente académicotecnológico con ponentes externos y sobre la base de complementos de formación, claramente reglados, editados por el MEC (1992); la tercera fase es de carácter más fenomenológico, no tan reglada, y se desarrolla a lo largo de los tres trimestres del curso con el objetivo básico del diseño, puesta en práctica y evaluación de una unidad didáctica por parte de cada grupo de profesores que se establezca. Por todo lo anterior, por lo menos en lo relativo al área de didáctica de las ciencias, estimamos que se crearon en torno a estos cursos un grupo selecto de profesionales cuyo pensamiento sobre la «acción docente» que desarrollan en sus aulas nos parece interesante analizar e incluso comparar con el de otros profesores que trabajen en sus mismos ámbitos profesionales.

\section{DESCRIPCIÓN DE LA INVESTIGACIÓN}

\section{Objetivos}

La parte del estudio que planteamos aquí se centra en dos objetivos:

- Analizar las posibles diferencias en las percepciones profesionales y en el pensamiento sobre «acción docente» del profesor de ciencias de educación secundaria según su experiencia y nivel de formación.

- Proponer líneas de actuación que contribuyan al desarrollo y la mejora de las trayectorias profesionales que puedan ser útiles para impulsar las ofertas de formación permanente del profesorado.

\section{Muestras}

Los sujetos investigados son profesores en activo relacionados con la docencia de las ciencias en la educación secundaria en la Comunidad de Madrid, con los cuales se constituyeron los siguientes grupos:

- De una población total de 127 profesores catalogados por haber realizado un curso ACD-A de ciencias en alguna de sus convocatorias, se localizó a los que aún permanecían en centros de la Comunidad en el curso 1996-97 (80 en total) y se les envió un cuestionario que devolvieron contestado $N=27$ profesores (que a partir de ahora les denominaremos ACD). De éstos se obtuvo una submuestra de profesores veteranos, con una media de veinticinco años de experiencia docente, de $n=11$ profesores que voluntariamente accedieron a que se les realizaran entrevistas y observaciones de aula.

- De otra población total de 798 profesores de ciencias, que estaban en activo en el curso 1996-97 en la Comunidad de Madrid y que no realizaron nunca el citado curso, se seleccionó a los que trabajaban en los centros de la demarcación del MEC «Madrid-Sur», muestra a la que se envió el cuestionario y que contestaron $N=76$ profesores (que referiremos como No-ACD). De ellos se obtuvo una submuestra de profesores consolidados profesionalmente, con una media de doce años de experiencia docente, de $n=9$ profesores que voluntariamente accedieron a que se les entrevistase y observase en el aula. La dificultad (no todo el mundo desea que entren en su clase) de encontrar una muestra representativa de profesores veteranos nos limitó, en este grupo, a la media de edad docente citada.

A la vista de la amplitud de datos que manejamos en nuestro trabajo (Martínez et al., 1997) aquí nos vamos a centrar fundamentalmente en el análisis de la información procedente del estudio de las respuestas a los cuestionarios pasados a toda la población estudiada de profesores en activo. El análisis de las entrevistas y observaciones de aula de las dos submuestras que acabamos de indicar puede consultarse en Rodrigo y otros (2000) y será utilizado para contrastar algunos de los resultados que se presentan en esta artículo.

\section{Instrumentos}

Como ya se ha indicado, las técnicas utilizadas han sido entrevistas, observaciones de aula y cuestionarios de opinión con el fin de conseguir una complementaridad de análisis. En este artículo nos referiremos especialmente a la última, es decir, al cuestionario, que pasamos seguidamente a describir (Anexo). Su versión definitiva se redactó a finales del curso 1995-96, previa consulta a expertos y tras la realización de un ensayo piloto que permitió poner a prueba el instrumento, reformular de nuevo algunos ítems y eliminar aquéllos poco discriminativos. En el instrumento se han considerado dos grandes bloques:

- El primero, centrado en dimensiones profesionales (Cuadro I), está estructurado en 8 preguntas de libre 
Cuadro I

Distribución de las proposiciones del cuestionario para la dimensión profesional.

\begin{tabular}{|c|c|c|}
\hline Dimensión profesional & Aspectos estudiados & Proposiciones del cuestionario \\
\hline Identificación & $\begin{array}{l}\text { - Edad, sexo, origen social } \\
\text { - Formación previa } \\
\text { - Motivos de elección profesional }\end{array}$ & $\begin{array}{l}\mathrm{a}, \mathrm{b}, \mathrm{c} \\
\mathrm{d} \\
\mathrm{g}\end{array}$ \\
\hline Percepciones profesionales & $\begin{array}{l}\text { - Modelos profesionales } \\
\text { - Satisfacción profesional } \\
\text { - Percepción de la formación inicial } \\
\text { - Percepción de la incidencia de factores sociales } \\
\text { y personales sobre el rendimiento escolar } \\
\text { - Percepción de las materias de ciencias }\end{array}$ & $\begin{array}{l}\text { e, f } \\
\text { h } \\
48,49 \\
50,51,52,55,56,57 \\
60,62 \\
53,61,63,64\end{array}$ \\
\hline
\end{tabular}

elección y 14 ítems cuyas posibles respuestas son: «totalmente de acuerdo», «de acuerdo», «indeciso», «en desacuerdo»y «totalmente en desacuerdo». Las preguntas de libre elección $(a-f)$, se pueden consultar en Martínez y otros (2001), y los contenidos de las dos restantes ( $g$ y $h$ ) se aprecian en el apartado de análisis de resultados (Tablas I y II respectivamente).

- El segundo está integrado por 45 declaraciones sobre la acción docente relativas a distintas dimensiones curriculares (Cuadro II) cuyas posibles respuestas son: «siempre», «frecuentemente», «a veces», «casi nunca»y «nunca».

En el bloque relativo a los aspectos curriculares y para determinar sus componentes, hemos adoptado los interrogantes genéricos «¿qué enseñar?», «¿qué, cómo y cuándo evaluar?» y «¿cómo enseñar?», que se hacen corresponder con: dimensión de contenidos, dimensión de evaluación y dimensión de metodología, respectivamente. En las proposiciones de este bloque se pretende contraponer dos tendencias: una concepción constructivista del proceso de enseñanza-aprendizaje (Flores et al., 2000; Porlán et al., 1998) que sustentaban los cursos ACD, frente a un enfoque más «reproductivo» o tradicional (véase el Anexo y la propuesta similar efectuada con declaraciones sobre el pensamiento docente en Martínez et al., 2001). Estas tendencias se han determinado al considerar que las concepciones o creencias que tienen los profesores presentan una cierta organización y dinámica que permitirían establecer o diferenciar modelos de actuación profesional. No obstante, en la realidad y tal como se desprende de este estudio, el profesorado se ajusta a modelos profesionales intermedios, como ya se sugieren en otros estudios (Fernández y Elortegui, 1996).

Cuadro II

Distribución de las proposiciones del cuestionario para las dimensiones curriculares.

\begin{tabular}{|c|c|c|}
\hline $\begin{array}{l}\text { Dimensiones } \\
\text { curriculares }\end{array}$ & Aspectos estudiados & Proposiciones del cuestionario \\
\hline Contenidos & $\begin{array}{l}\text { - Relación con otros conocimientos } \\
\text { - Fuentes y organización }\end{array}$ & $\begin{array}{l}34,35,36,37,38,39 \\
40,41,42,43,44,45\end{array}$ \\
\hline Evaluación & $\begin{array}{l}\text { - Instrumentos } \\
\text { - Diseño y organización } \\
\text { - Finalidad }\end{array}$ & $\begin{array}{l}1,2,3,4 \\
5,6,7,9,11 \\
8,10\end{array}$ \\
\hline Metodología & $\begin{array}{l}\text { - Planificación } \\
\text { - Desarrollo de la enseñanza } \\
\text { - Adaptación al alumno } \\
\text { - Motivación/Participación } \\
\text { - Recursos }\end{array}$ & $\begin{array}{l}16,17,29 \\
12,18,20,21,22 \\
13,24,25 \\
14,15,19,23,27,28 \\
30,31,32,33\end{array}$ \\
\hline
\end{tabular}


La información recogida se ha analizado mediante dos procedimientos. En las preguntas diseñadas como de libre elección se ha realizado un recuento para cada contestación, con un cálculo del porcentaje relativo de cada respuesta frente al total. El análisis comparativo de los datos obtenidos de las cuestiones con escala tipo Lickert se ha llevado a cabo mediante el estadístico de la $t$ de student con un nivel de significatividad de 0,05.

\section{ANÁLISIS DE RESULTADOS Y CONCLU- SIONES}

De acuerdo con la estructura del cuestionario utilizado en la investigación, vamos a presentar los resultados según las dimensiones definidas para su diseño. En primer lugar, va a aparecer la información correspondiente a las características personales y profesionales de la muestra estudiada indicándose a continuación todo lo relativo a sus opiniones en el campo de las dimensiones curriculares.

\section{Dimensiones profesionales}

\section{Identificación}

De los datos extraídos de los cuestionarios con relación a la composición por edad, sobresale que la muestra ACD está en el grupo modal entre 41 y 45 años y la noACD entre 36 y 40 años. Tal diferencia es explicable por la utilización del criterio administrativo de antigüedad a la hora de seleccionar a los profesores que integraban los diferentes cursos ACD, de tal manera que, mientras el grupo menos experimentado tiene una media de edad ( 38 años) similar a la de todo el colectivo docente español de enseñanza secundaria, el grupo más veterano aumenta esa media de edad en cuatro años (42 años).

Respecto a los orígenes sociales, cabe destacar que del orden del $80 \%$ del profesorado pertenece a la denominada clase media. De la muestra ACD, el 29,6\% es de clase media-alta, integrada en mayor medida por sectores de la pequeña burguesía, frente al 19,7 \% para el grupo noACD. En contraposición, un $28,9 \%$ de este último colectivo presenta una extracción social más baja procedente de clase media no propietaria (empleados, funcionarios...) que sólo alcanza el 14,8\% para los ACD. Por otra parte hemos de indicar que la formación inicial de ambos colectivos es muy similar, con el predominio de licenciaturas en química y en biología.

Los motivos de elección profesional presentan una gran semejanza entre las muestras (Tabla I). Así, se destacan, en la población estudiada, los aspectos de insatisfacción de tipo extrínsecos relativos a «las condiciones de trabajo, la valoración social y las perspectivas de promoción», consideraciones que concuerdan con lo planteado en estudios con profesores de niveles no universitarios realizados por distintos autores en España (Pérez Gómez y Gimeno, 1992; Guerrero, 1997).

\section{Percepciones profesionales}

En general aparecen diferencias en el modelo de profesionalidad por el que optan los sujetos estudiados (Tabla II). Así, utilizando la terminología acuñada por Broadfoot y Osborn (1988), los profesores ACD mani-

\begin{tabular}{|l|c|c|}
\hline Motivos & $\begin{array}{c}\text { Respuestas de la } \\
\text { muestra ACD } \\
\mathbf{\%}\end{array}$ & $\begin{array}{c}\text { Respuestas de la } \\
\text { muestra no-ACD } \\
\text { \% }\end{array}$ \\
\hline Extrínsecos & $\mathbf{6 5 , 4}$ & $\mathbf{6 1 , 4}$ \\
\hline Coyuntura del mercado laboral & 51,2 & 45,1 \\
\hline Disponibilidad de tiempo libre & 6,3 & 10,9 \\
\hline Facilidad de acceso & 6,2 & 4,3 \\
\hline Imagen social & 1,6 & 1,1 \\
\hline Intrínsecos & $\mathbf{3 4 , 6}$ & $\mathbf{3 8 , 6}$ \\
\hline Vocación & 23,6 & 26,4 \\
\hline Servicio a la comunidad & 4,7 & 8,1 \\
\hline Tradición familiar & 6,3 & 3,3 \\
\hline
\end{tabular}


Tabla II

Contextos que influyen en el trabajo docente (las cifras aparecen en \% y la diferencia hasta 100 en cada casilla corresponde a «no saben»o «no contestan»).

\begin{tabular}{|c|c|c|c|c|c|c|}
\hline \multirow{2}{*}{$\begin{array}{l}\text { Grado de } \\
\text { influencia }\end{array}$} & \multicolumn{2}{|c|}{$\begin{array}{l}\text { Positiva } \\
(\%)\end{array}$} & \multicolumn{2}{|c|}{$\underset{(\%)}{\text { Ninguna }}$} & \multicolumn{2}{|c|}{$\begin{array}{c}\text { Negativa } \\
(\%)\end{array}$} \\
\hline & $\mathrm{ACD}$ & NO-ACD & $\mathrm{ACD}$ & NO-ACD & $\mathrm{ACD}$ & NO-ACD \\
\hline Responsabilidad profesional & 96,3 & 94,7 & 0,0 & 0,0 & 0,0 & 1,3 \\
\hline Alumnos & 92,6 & 86,9 & 0,0 & 3,9 & 7,4 & 3,9 \\
\hline Compañeros de departamento & $\mathbf{7 7 , 7}$ & 89,5 & 11,1 & 5,3 & 7,5 & 1,4 \\
\hline Libros de texto & $\mathbf{7 4 , 0}$ & 82,9 & 14,8 & 6,6 & 7,6 & 7,8 \\
\hline Reciclaje & 96,3 & 71,0 & 0,0 & 22,4 & 3,7 & 3,8 \\
\hline Claustro & 63,0 & 67,1 & 18,5 & 21,1 & 14,8 & 6,4 \\
\hline Programas oficiales & 25,9 & 35,5 & 18,4 & 28,9 & 48,1 & 30,2 \\
\hline Dirección & 40,7 & 38,2 & 44,4 & 46,1 & 3,7 & 10,5 \\
\hline Padres & 44,4 & 35,5 & 0,0 & $\mathbf{5 3 , 9}$ & 51,9 & 6,5 \\
\hline Inspección & 3,7 & 9,2 & 77,8 & 60,5 & 18,5 & 25,0 \\
\hline Consejo escolar & 22,2 & 26,3 & 74,1 & 69,7 & 3,7 & 1,3 \\
\hline
\end{tabular}

fiestan una profesionalidad más extensa, con un compromiso mayor con aspectos más allá de su trabajo en el aula (padres, programas oficiales, reciclaje). En la muestra no-ACD, la profesionalidad está más ligada a lo intrínseco de la práctica docente (el trabajo en el aula). Con referencia a lo anterior, en las entrevistas personales realizadas a una parte de los profesores se pone de manifiesto, por ejemplo, que la mayoría de los profesores ACD dice que forma parte de grupos de trabajo didáctico, ajenas a su centro escolar, algo que sólo hace la mitad de los otros profesores (Rodrigo et al., 2000).

En términos generales, aquí aparece reflejada una percepción profesional muy limitada, a nuestro juicio, en uno y otro colectivo. Por ello, creemos que sería necesario definir los rasgos de profesionalidad que caracterizan el trabajo docente y que esta conciencia profesional se debería introducir, desde la formación inicial, y profundizar, en la formación permanente.

Por otra parte, la satisfacción profesional de la muestra ACD es mayor que la de la no-ACD (Tabla III). En términos numéricos, la media es del $68,2 \%$ para los primeros y del $54,7 \%$ para los segundos. Desde el punto de vista cualitativo, los profesores ACD se muestran satisfechos en todos los aspectos encuestados salvo en los casos de la ratio de alumnos por clase, el currículo de ciencias de la ESO, la valoración social y las expectativas de promoción. Los profesores no-ACD ligan la insatisfacción profesional a los mismos aspectos que sus colegas y, además, redundando en los aspectos relativos a la actitud de padres y alumnos, a aspectos retributivos y a aspectos referidos a los nuevos currículos, siendo en este sentido, más proclives para el cumplimiento ritua- lista de la profesión y para el burn-out (Freechman, 1988). Estos datos relativos a la motivación-satisfacción coinciden plenamente con lo puesto de manifiesto en las entrevistas personales realizadas en las submuestras de la población estudiada (Rodrigo et al., 2000).

En resumen, los profesores perciben que su tarea social está poco valorada, pero esta circunstancia parece paliarse por los supuestos beneficios de las vacaciones y la autonomía en el desempeño de su actividad. Aquí de nuevo podemos reflexionar sobre la necesidad de generar y potenciar cauces de profesionalización y control de la actividad para potenciar las motivaciones profesionales.

En relación con las percepciones sobre la incidencia de factores sociales y personales en el rendimiento escolar, la formación inicial y sobre las materias de ciencias impartidas, sólo se aprecian diferencias significativas entre los dos grupos de profesores estudiados en las respuestas efectuadas en los ítems 48, 53 y 57 (Gráfico 1). Los profesores ACD muestran en mayor medida la insatisfacción en cuanto a la formación científica recibida en la universidad, algo que se corrobora con los resultados de las entrevistas realizadas a la submuestra correspondiente en la que se solicita una formación más acorde con la proyección didáctica de las diferentes disciplinas (Rodrigo et al., 2000). Además, este colectivo relativiza en mayor grado la aptitud de los alumnos como requisito para sus materias y se muestra más inclinado a considerar la importancia de los métodos utilizados en la enseñanza como un factor importante en el rendimiento del alumno, hecho que también se destaca en las entrevistas. Por otro lado, los profesores no-ACD 
manifiestan posturas más tradicionales en los aspectos indicados.

Dentro de las semejanzas en las afirmaciones de ambos grupos podemos destacar como alentador que existan acuerdos sobre: $a$ ) la necesidad de integrar una formación psicopedagógica en la formación inicial (ítem 49), idea corroborada en las entrevistas; $b$ ) la consideración de que la ciencia potencia el pensamiento crítico (ítem $63)$; c) que los chicos y las chicas tienen una aptitud similar en el aprendizaje de las ciencias (ítem 52).

Tabla III

Satisfacción profesional del profesorado de ciencias estudiado (ACD, $N=27$ y no-ACD, $N=76$ donde la diferencia en cada casilla hasta el $100 \%$ corresponde a «no sabe»o «no contesta»).

\begin{tabular}{|l|cc|}
\hline \multirow{2}{*}{ Motivos } & \multicolumn{2}{|c|}{ Satisfacción (\%) } \\
\cline { 2 - 3 } & ACD & No-ACD \\
\hline Vacaciones & 100,0 & 98,7 \\
\hline Autonomía en aula & 100,0 & 97,3 \\
\hline Horario lectivo & 96,3 & 85,5 \\
\hline Actitud alumnos & 77,8 & 32,9 \\
\hline Actitud padres & 74,2 & 39,5 \\
\hline Resultados de su labor & 74,1 & 52,6 \\
\hline Recursos disponibles & 70,4 & 50,0 \\
\hline Ambiente del centro & 66,7 & 78,9 \\
\hline Retribuciones & 55,6 & 31,6 \\
\hline Ratio alumnado & 37,0 & 19,7 \\
\hline $\begin{array}{l}\text { Currículo de ciencias } \\
\text { en la ESO }\end{array}$ & 33,4 & 15,8 \\
\hline Valoración social & 29,6 & 15,7 \\
\hline Perspectivas de promoción & 7,4 & 14,5 \\
\hline
\end{tabular}

En relación con la declaración 55 sobre si el éxito o el fracaso del alumnado depende básicamente de sus características personales, la totalidad de los profesores se muestran indecisos. Esta duda desaparece en las entrevistas, en las que todos ellos manifiestan que la responsabilidad ante el fracaso del alumno es «extrínseca» al profesor, según un sesgo generalizado de autodefensa (Clark y Peterson, 1986; Kelley, 1972). También existe indecisión, en ambas muestras de profesores, ante la cuestión 61 en la cual se achaca la dificultad de las materias científicas al lenguaje técnico, impresión que se mantiene en las entrevistas.

Con relación al resto de los ítems de esta dimensión, hay que incidir en que para la totalidad del profesorado, no hay diferencias atribuidas al sexo del alumnado en cuan- to a su capacidad para el aprendizaje. Hay una cierta indecisión sobre el grado de influencia del origen social en el aprendizaje y en la disponibilidad de recursos así como en la influencia del desarrollo cognitivo de los individuos en el rendimiento escolar. También se aprecian dudas sobre la importancia de la personalidad y actitud del profesorado, dudas que se despejan en las entrevistas en las que, frecuentemente, los profesores han matizado su pensamiento aclarándonos que para ellos un buen profesor debe tener ciertas cualidades personales, lo cual abriría el debate sobre la selección previa de los estudiantes con «vocación» de docentes, aspecto que también resalta Esteve (1997).

\section{Dimensiones curriculares}

En este apartado se presenta el análisis de resultados y las conclusiones correspondientes a la «acción docente».

\section{Dimensión de contenidos}

El análisis comparativo de las contestaciones de las muestras de profesores a las preguntas del cuestionario correspondientes a esta dimensión aparecen en el gráfico 2 . Aquí se pueden detectar diferencias de opinión en «la tendencia a considerar el conocimiento escolar como conocimiento científico actualizado, objetivo y correcto» (ítem 36), en la «consideración de las ideas de los alumnos como errores» (ítem 38 ) y en la «no-utilización de fuentes diversas para la selección de contenidos» (ítem 41). Estas proposiciones son más asumidas por parte de la muestra no-ACD, y están dentro de la tendencia que hemos considerado como tradicional, situándose la muestra ACD en posiciones más constructivistas en la temática a que hacen referencia estos ítems.

Las diferencias apreciadas se han matizado con las observaciones de aula efectuadas en el sentido de que, a mayor experiencia y formación (grupo ACD), se tiende a utilizar más variedad de recursos en la acción de aula (Rodrigo et al., 2000); una conclusión similar, a la que plantea Barquín (1991) al estudiar el comportamiento de profesores veteranos. Por el contrario, en las observaciones de aula de los profesores no-ACD se pone de manifiesto que, mayoritariamente, los contenidos que se imparten proceden, salvo excepciones, exclusivamente del libro de texto, utilizándose una secuencia lógica de la materia frente a la psicológica o adaptada al alumno. Esto también se destaca en estudios con profesores poco experimentados de bachillerato, como los efectuados por Pérez Gómez y Gimeno (1992).

Es de señalar que, en cuestiones más ligadas a la práctica escolar, no se aprecian diferencias significativas entre las muestras; ambas se manifiestan muy conservadoras a la hora de organizar y determinar el nivel de contenidos que se explican en clase. Esto se comprueba en las respuestas al ítem 42, «proponen una secuencia lineal de temas para organizar los contenidos escolares»; al ítem 43, «utilizan poco la organización de contenidos en 
Gráfico 1

Comparación entre las muestras para la dimensión profesional.

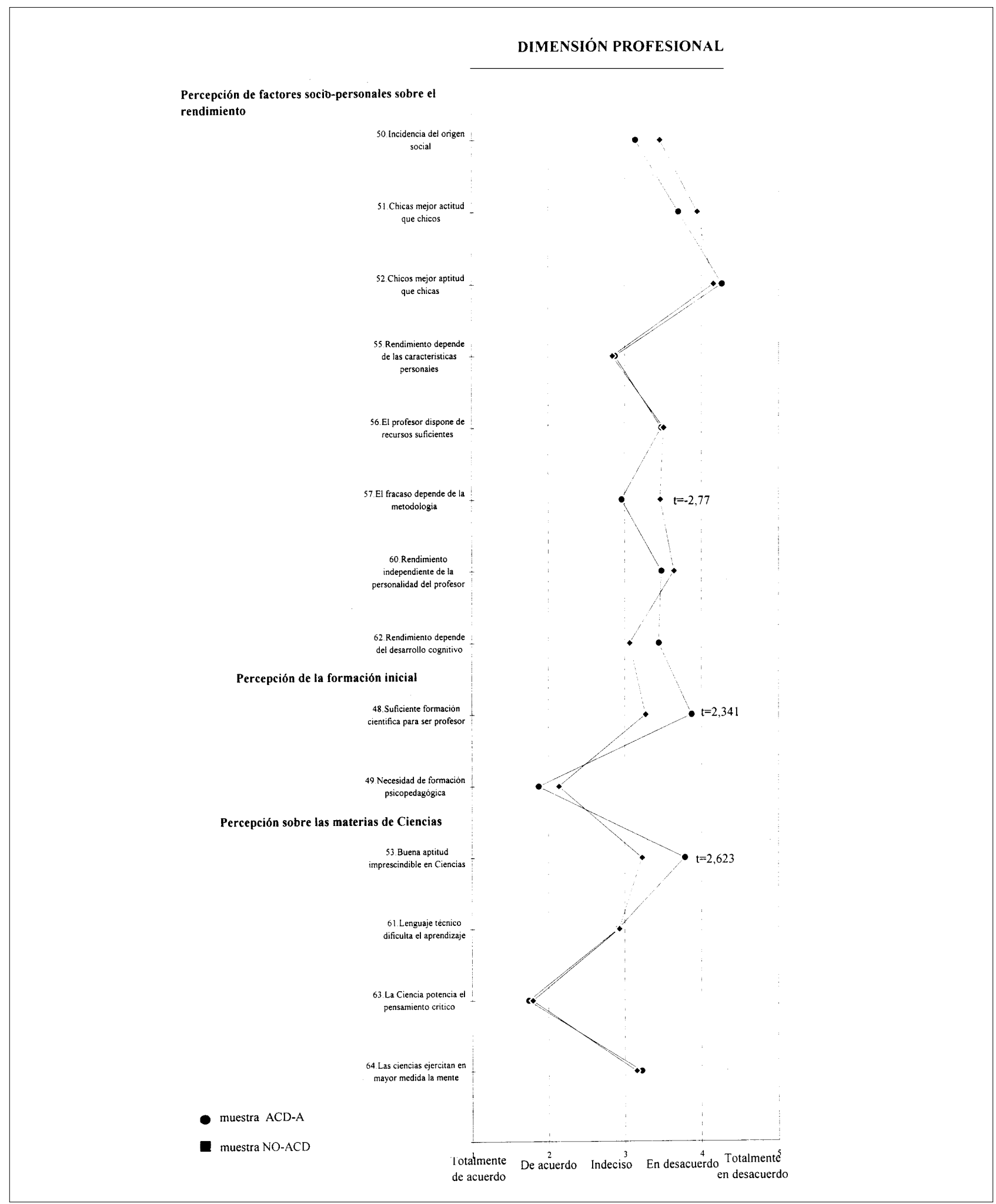


mapas conceptuales»; y al ítem 44, «el nivel de conocimiento al que tienen que llegar los alumnos es el prefijado en la programación».

Por último, se debe valorar positivamente que todos los profesores afirmen que frecuentemente «los contenidos de trabajo con los alumnos tratan aspectos relevantes para la vida cotidiana y la integración social de las personas» (ítem 35) e «introducen cuestiones históricas para poner de manifiesto el carácter evolutivo del conocimiento científico» (ítem 37), práctica que representa una mejora a la hora de seleccionar contenidos.

Merece la pena indicar la inconsistencia que aparece entre las respuestas al ítem 44, ya mencionado, y al 45 en que los profesores afirman que, con mucha frecuencia evalúan positivamente a los alumnos cuando han experimentado una evolución favorable aunque no hayan alcanzado el nivel esperado. Esta contradicción no debe sorprender, ya que el procedimiento de calificar a los alumnos es uno de los temas que genera mayor polémica entre los profesionales de la educación.

\section{Dimensión de evaluación}

Como se muestra en el gráfico 3 , se han encontrado diferencias significativas en las declaraciones $2,6,7$ y 8 , que corresponden a las categorías de: Instrumentos («no se utilizan instrumentos para evaluar actitudes», ítem 2), Diseño y organización («pocas veces se analizan los resultados de la evaluación de los alumnos con los compañeros del departamento y se revisan con éstos la programación de las asignaturas», ítems 6 y 7) y Finalidad («en muy pocas ocasiones evalúan para comprobar sólo el nivel de conocimientos previsto», ítem 8). Cabe destacar que estas diferencias muestran que los profesores del grupo ACD reflejan posturas más tradicionales que las presentadas por el otro colectivo de profesores, salvo en lo correspondiente a la finalidad de la evaluación, en que se aprecia una perspectiva más evolucionada.

Por otra parte, hay gran consenso entre todos los profesores estudiados en planteamientos claramente tradicionales en relación con la idea de evaluar fundamentalmente con exámenes escritos (ítem 1) y de preparar las pruebas de evaluación siguiendo criterios propios (ítem 5). En términos generales, hemos apreciado que el profesorado más preparado padece lo que podríamos llamar la soledad del veterano, que en muchos casos le lleva a no compartir sus experiencias e ideas con el resto de compañeros menos preparados de su entorno próximo.

Para terminar con este análisis comparativo, es destacable que existe también un acuerdo bastante unánime y positivo en la utilización de la calificación del trabajo de laboratorio para evaluar (ítem 3) y en el empleo de la evaluación para informar y orientar a los alumnos en sus dificultades (ítems 9 y 10).

Los resultados presentados se han contrastado con las observaciones de aula en las que, además, se ha aprecia- do que los profesores investigados tienen mayoritariamente en cuenta para la evaluación el trabajo que el alumno realiza en el aula (Rodrigo et al., 2000). Estos resultados unidos a los del cuestionario señalan la necesidad de profundizar en esta dimensión curricular cuando se diseñan programas de formación docente, conclusiones a las que también llegan autores como Pérez Gómez y Gimeno (1992) al estudiar el pensamiento pedagógico de profesores de bachillerato de distintas especialidades.

\section{Dimensión de metodología}

De la información presentada en la gráfico 4 , se aprecia que existen diferencias significativas entre las dos muestras en las categorías y respuestas a las cuestiones: Planificación (ítems 12 y 17), Desarrollo de la enseñan$z a$ (ítems 20 y 22), Adaptación al alumno (ítems 24 y 25), Motivación/Participación (ítems 14, 23 y 28) y en la categoría de Recursos (ítem 32).

En lo referente a la planificación de la enseñanza, destaca como positivo que, frecuentemente, todos los profesores investigados admiten la «importancia de consultar las ideas previas con que los alumnos se enfrentan a la enseñanza» (ítem 16). No obstante, ambos colectivos estudiados discreparían en cómo llevar a efecto la acción docente: si en unidades de trabajo didáctico estructuradas o «ciñéndose exclusivamente al libro de texto y a la explicación verbal» (ítems 12 y 17). Precisamente, las posturas definidas por los profesores más maduros (ACD) parecen más congruentes y evolucionadas en estos aspectos que en el caso de los profesores con menos experiencia. Igualmente ocurre, ya dentro de la categoría de Desarrollo de la enseñanza, al considerar el «sentido de las prácticas como comprobación de la teoría dada» (ítem 22) o al preguntar sobre «la inclusión de las implicaciones sociales de lo que se enseña» (ítem 20 ), aspectos en los que los profesores no-ACD siguen manteniendo posturas más tradicionales. Sin embargo, es positiva la manifestación realizada por todo el colectivo de «permitir las iniciativas de los alumnos en las aulas» (ítem 13) o «la inclusión de problemas cotidianos en el desarrollo de la docencia» (ítem 21).

En cuanto a la adaptación al alumno, los resultados ponen en evidencia que el profesorado se muestra claramente indeciso al definirse sobre la «atención a la diversidad» (ítems 24 y 25). Estas respuestas son reflejo de la complejidad de una temática que es difícil de resolver en la realidad escolar, lo que explica las manifestaciones de la mayoría del profesorado consultado. Esto pone de relieve la falta de preparación del profesorado en ciertos aspectos, que hasta se podría cuestionar si son de su incumbencia o de otros sectores de la sociedad.

Ya dentro de la categoría de Motivación/participación y teniendo en cuenta la opinión de los encuestados sobre «la inclusión de pequeñas investigaciones en la marcha de clase» (ítem 14) y de la oportunidad de que los alumnos trabajen en grupo (ítem 23), hemos de destacar que los profesores ACD están más actualizados que sus 
Gráfico 2

Comparación entre las muestras para la dimensión de contenidos.

\section{DIMENSIÓN DE CONTENIDOS}

Relación con otros conocimientos

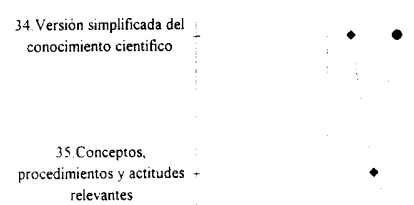

36 Version actual de:

conocimiento cientifico

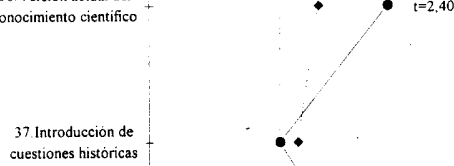

tiones historicas

38. Sustitución ideas

38. Sustitución ideas
erróneas alumno por - -

correctas
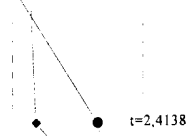

$i=2,4138$

Fuentes y organización de los contenidos

39. Conocimiento alternativo al cientifico y$$
\text { escolar }
$$

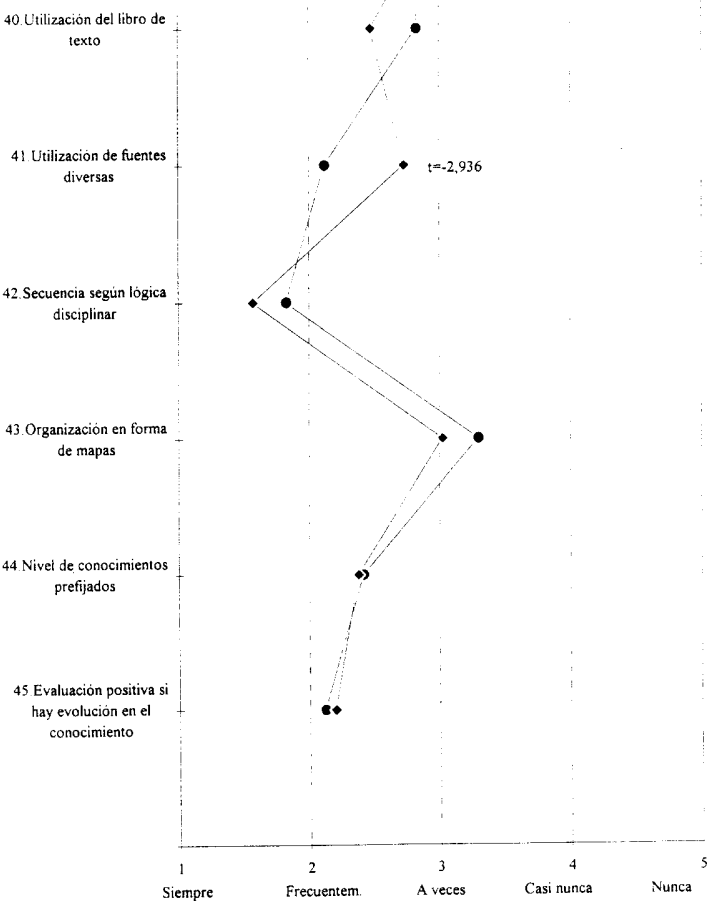


Gráfico 3

Comparación entre las muestras para la dimensión de evaluación.

\section{DIMENSIÓN DE EVALUACIÓN}

Instrumentos

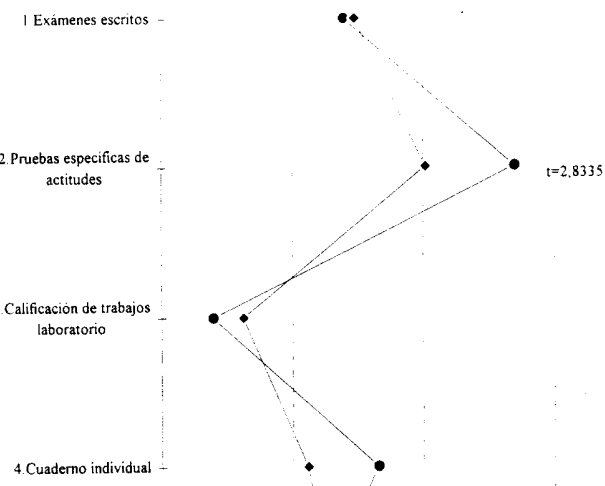

Diseño y organización

- muestra ACD-A

- muestra NO-ACD

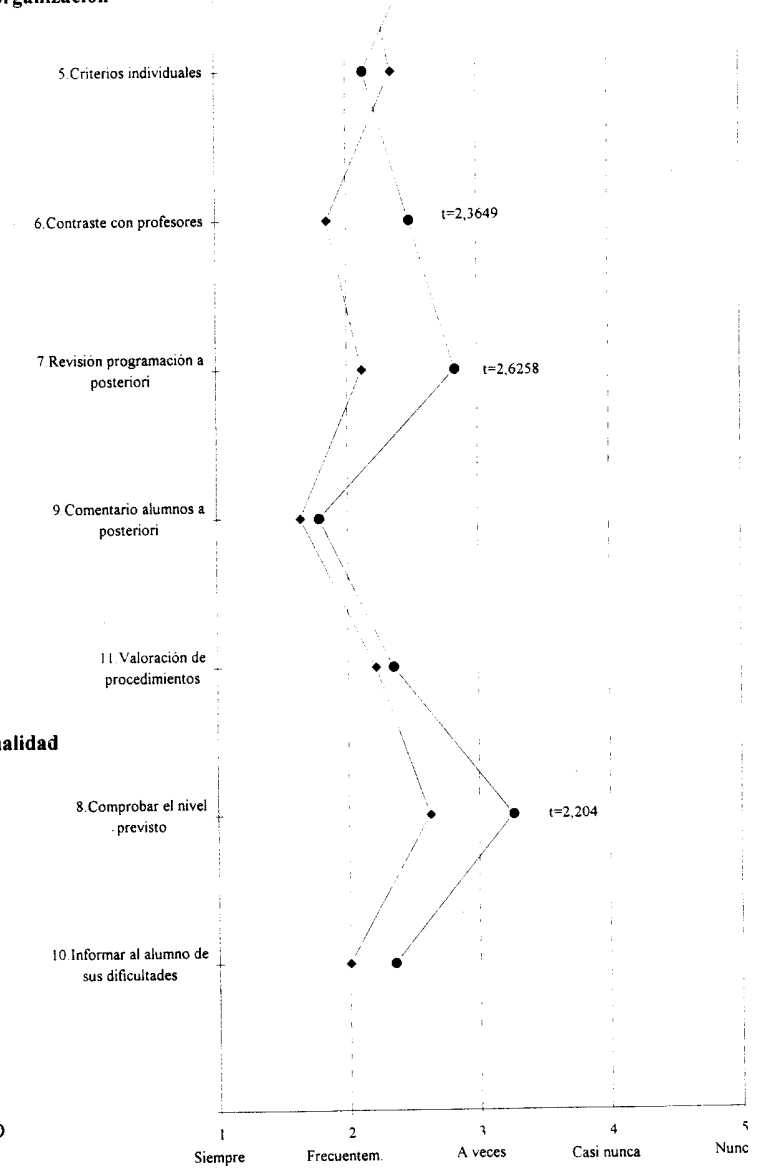


Gráfico 4

Comparación entre las muestras para la dimensión de metodología.

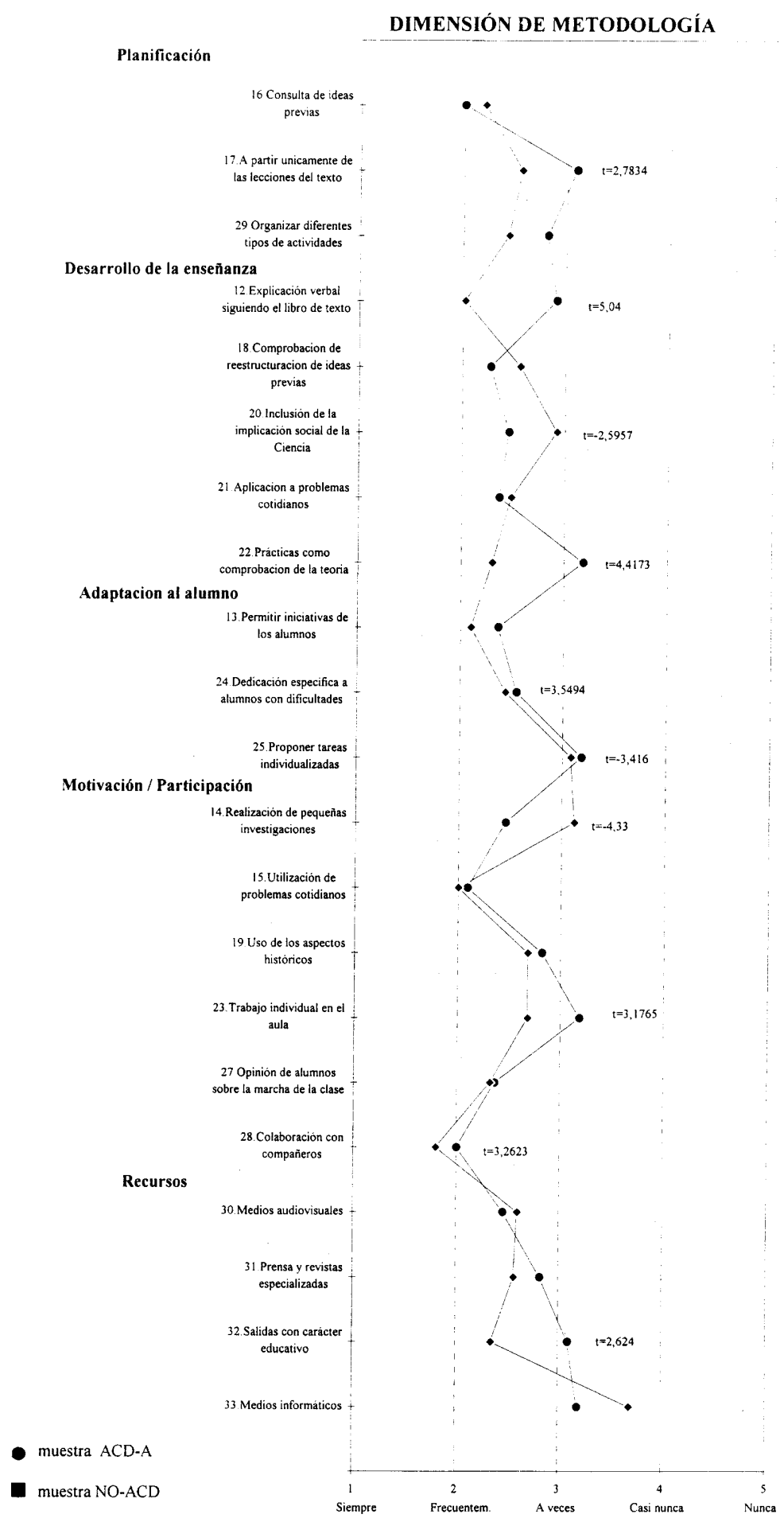


colegas, los cuales, sin embargo, se muestran más proclives a «la colaboración con compañeros del departamento» (ítem 28) (aquí aparece de nuevo otro indicador de lo que hemos llamado soledad del veterano). Por otra parte, se detecta un acuerdo bastante generalizado sobre «motivar a los alumnos, empleando problemas cotidianos como recurso para enseñar ciencia» (ítem 15), aspecto que en la actualidad se considera básico para que el alumnado aprenda.

Por último, en cuanto a la categoría de Recursos, se constata una cierta uniformidad de pensamiento entre todo el profesorado investigado salvo en lo referente a «las salidas fuera del aula con fines educativos» (ítem 32), que es más cuestionado por los profesores más veteranos. Queremos destacar la poca utilización de medios informáticos por el conjunto de encuestados, aspecto que debería ser revisado en los cursos de formación permanente por su transcendencia para el desarrollo futuro de la enseñanza en la sociedad del siglo XxI.

Con los datos procedentes de las observaciones de aula podemos matizar algunos de los aspectos comentados anteriormente; así, la utilización de variedad de materiales para realizar su labor educativa (muchos de ellos de propia elaboración) parece que es una característica muy presente en los profesores ACD estudiados, mientras que la acción docente del profesorado menos formado se suele ligar al libro de texto y a la resolución de las tareas que en él se incluyen (Rodrigo et al., 2000). Cabe señalar que en el desarrollo de las actividades de aula se ha observado que los alumnos trabajan en grupo indistintamente según sea el tipo de profesor, realidad contraria a los resultados obtenidos con los cuestionarios.

Como síntesis general y tomando como referencia que el desarrollo del conocimiento profesional se concibe como un proceso de cambio gradual, cabría esperar -y así se confirma en dos de las dimensiones estudiadas como son la de metodología y evaluación- una gran heterogeneidad de situaciones en el pensamiento de acción docente manifestado por los profesores investigados. No obstante, se suele dar una mayor madurez de planteamientos docentes en los profesores más formados (ACD) y, especialmente en la dimensión de contenidos, se establece una mayor segregación entre la forma de pensar del profesorado atendiendo al nivel de experiencia y formación. Lo anterior vendría a reforzar la importancia que tiene una formación permanente que supere unos mínimos de calidad contrastada.

\section{IMPLICACIONES PARA LA FORMACIÓN}

En este apartado, tomando como referencia los resultados y las conclusiones que se han aportado a lo largo del artículo, vamos a proponer algunas ideas a tener en cuenta en los programas de formación. Queremos destacar que nuestra propuesta para la formación permanente recoge aspectos que mayoritariamente estaban presentes en los cursos ACD de nivel A y que han sido asumidos en su mayor parte por el grupo que asistió a ellos, lo cual se convierte en un resultado objetivo de este tipo de formación.

\section{Dimensiones del plano profesional}

En este campo, la superación de los condicionantes extrínsecos de la carrera docente, tales como las perspectivas de promoción, la valoración social, las condiciones de trabajo... podría ser una condición coadyuvante para lograr una mejora en el clima de trabajo en los centros, creando una conciencia de profesionalidad en los docentes. Esta profesionalización debería promover, en los cursos de formación, y a partir de la reflexión y el debate, la responsabilidad social de los profesores dentro de la comunidad escolar.

En el ámbito de las percepciones del profesorado sobre su interacción con los alumnos, y a la luz de nuestros estudios, habría que:

a) trabajar propuestas de cambio de mentalidad con respecto a la atribución del fracaso escolar; asunto del que los profesores no llegan a sentirse responsables, adoptando lo que autores como Clark y Peterson (1986) denominaron actitudes autodefensivas;

b) en línea con lo anterior, una clave podría ser ir superando la concepción frecuente entre el profesorado de que las materias de ciencias son difíciles y abstractas y que, por lo tanto, el éxito escolar depende en gran medida de las características personales del alumnado.

Por último, en relación a cómo perciben los profesores su formación, queremos destacar aquí que un pensamiento ampliamente extendido entre el profesorado investigado es que la formación «sea realista y ligada a la situación de las aulas», circunstancia que también ponen de manifiesto distintos autores (Porlán et al., 1998; Mellado et al., 1997). Para ello, creemos que la formación inicial debe favorecer que el profesorado adquiera un sólido conocimiento psicopedagógico tan necesario para resolver situaciones de aula y que, durante la formación permanente, se debería potenciar la constitución de grupos de trabajo fundamentalmente ligados a centros escolares o al menos ubicados en la zona donde los profesores desarrollan su labor docente.

Por último y, como fruto de las ideas recogidas, podemos indicar que sería necesario abordar, de forma sistemática, aspectos epistemológicos e históricos en los estudios universitarios de ciencias, temas que hoy en día son colaterales pero cuya exclusión suponen un handicap para los futuros profesores y profesionales en general (Abd-El-Khalick y Lederman, 2000).

\section{Dimensiones curriculares}

Dimensión de contenidos

Las consideraciones teóricas sobre la naturaleza del conocimiento escolar, su reconocimiento como una for- 
ma peculiar de conocimiento, las relaciones con el conocimiento científico y el de los alumnos -en definitiva, las cuestiones más epistemológicas- parecen necesitar de un mayor tratamiento en la formación permanente del profesorado, ya que incluso los profesores más formados parecen requerir una mayor profundización en estos aspectos (Jiménez, 1995).

En relación con los contenidos curriculares, habría que profundizar en las cuestiones relacionadas con la transposición didáctica y la importancia de conocer y utilizar las «ideas de los alumnos» a la hora de seleccionar los objetivos educativos y diseñar actividades de aula. En concreto, a la hora de seleccionar y secuenciar los contenidos escolares, se debería insistir en el «saber hacer» (análisis didáctico de contenidos escolares concretos utilizando diversidad de fuentes, libros de texto, ideas previas, mapas conceptuales...) más que en la teorización de este planteamiento.

\section{Dimensión de evaluación}

Esta dimensión se manifiesta como un campo de especial interés para mejorar en los procesos de formación. Como actuación prioritaria, de forma integrada se deberían diseñar instrumentos de evaluación ligados a situaciones reales de aula que abarquen conocimientos conceptuales, de procedimiento y de actitud y que permitan una individualización del proceso de enseñanzaaprendizaje. Esta necesidad ha sido puesta en evidencia en otros estudios, tanto con profesores de educación secundaria en activo (Brincones et al., 1986) como de formación inicial (Pérez Gómez y Gimeno, 1992; Martínez et al., 2001).

Además, en la línea de crear espacios de coordinación del trabajo de los profesores, se debe insistir en el diseño colegiado de los instrumentos de evaluación, es decir, en la participación de todos los miembros del departamento didáctico.

\section{Dimensión de metodología}

A la luz del estudio realizado, sería necesario fomentar el diseño de materiales y recursos para la acción didáctica. En este sentido es de vital importancia llevar a cabo esta actividad formativa por tutores expertos y con el estudio de casos reales.
En relación con el punto anterior, en el marco de procesos de formación permanente, habría que introducir módulos de discusión y debate sobre los resultados obtenidos al introducir, en las clases, materiales didácticos no exclusivamente centrados en los libros de texto, aspecto también reclamado por otros autores (De Pro, 1995).

Por último, una temática a desarrollar durante los cursos de formación sería la relativa a la adaptación a la diversidad dentro de las aulas, dada la heterogeneidad del alumnado y además parece impensable suponer que esta situación evolucione hacia grupos de clase más homogéneos.

Este aspecto se manifiesta como preocupación permanente entre la mayoría del profesorado investigado, lo cual pone de manifiesto que los programas de formación no han abordado con eficacia esta problemática. Como pauta de trabajo podría ser adecuado tratar de resolver casos reales que presenten los integrantes de los grupos de trabajo y que deberían surgir de las programaciones que estén desarrollando, en sus respectivos centros, los participantes en los cursos. Basándose en la especificidad de las materias de ciencias, tal como hemos percibido en algunas aulas, el hecho de utilizar las actividades prácticas de aula o de laboratorio, así como los trabajos en pequeños grupos, podría dar origen a una diversificación de tareas escolares muy fructíferas (Rodrigo et al., 2000).

No queremos terminar sin retomar la idea del desarrollo profesional, que pasa por una formación inicial de calidad integradora de saberes científico-didácticos y una formación permanente que debe planificarse, por una parte, sobre la base de las necesidades profesionales en general y, por otra, que se deberá adaptar a las necesidades y peculiaridades concretas de cada centro y de los grupos de profesores (Martínez et al., 2001; Mellado, 1998; De Jong, 1998).

Finalmente, queremos indicar que todas estas reflexiones y propuestas no podríamos haberlas realizado sin la colaboración desinteresada de los profesores que nos han cedido parte de su tiempo y nos han mostrado sus maneras de pensar y actuar. Desde aquí queremos manifestarles nuestro agradecimiento más sincero. 


\section{REFERENCIAS BIBLIOGRÁFICAS}

ABD-EL-KHALICK, F. y LEDERMAN, N.G. (2000). Improving science teachers' conceptions of nature of science: a critical review of the literature. International Journal of Science Education, 22(7), pp. 665-701.

BARQUÍN, J. (1991). La evolución del pensamiento pedagógico del profesor. Revista de Educación, 294, pp. 245-274.

BRINCONES, I., FUENTES, A., NIEDA, J. PALACIOS, M.J. y OTERO, J. (1986). Identificación de comportamientos deseables del profesorado de ciencias experimentales del bachillerato. Enseñanza de las Ciencias, 4(3), pp. 209-222.

BROADFOOT, P. y OSBORNO, M. (1988). What professional responsibility means to teachers: national contexts and classrooms constants. British Journal of Sociology of Education, 9(3), pp. 265-287.

CLARK, C. M. y PETERSON, P. L.(1986). Teachers' Thought Processes, en Whitrock (ed.). Handbook of Research in Teaching. Nueva York: Macmillan.

DE JONG, O., KORTHAGEN, F. y WUBBELS, T. (1998). Research on science teacher education in Europe: teacher thinking and conceptual change, en Fraser, B.J. y Tobin, K.G. (eds.). International Handbook of Science Education, pp. 745-758. Gran Bretaña: Kluwer Academic Publishers.

DE PRO, A. (1995). Formación de profesor de secundaria versus profesor tutor de prácticas de secundaria, en Blanco, L.J. y Mellado, V. (coords.). La formación del profesorado de ciencias y matemáticas en España y Portugal, pp. 375-397. Badajoz: Universidad de Extremadura.

ESTEVE, J.M. (1997). La Formación inicial de los profesores de secundaria. Barcelona: Ariel.

FERNÁNDEZ, J. y ELORTEGUI, N. (1996). ¿Qué piensan los profesores acerca de cómo se debe enseñar? Enseñanza de las Ciencias, 14(3), pp. 331-342.

FLORES, F., LÓPEZ, A., GALLEGOS, L. y BAROJAS, J. (2000). Transforming science and learning of physics teachers. International Journal of Science Education, 22(2), pp. 197-208.

FREECHMAN, S. (1988). Teachers' burn-out and institutional stress, en Ozga, J.Schoolwork. Milton Keynes: Open University Press.

GUERRERO SERÓN, A. (1997). El perfil socioprofesional del profesorado de media y su actitud ante la reforma de la enseñanza secundaria. Revista de Educación, 314, pp. $247-$ 266.

GUERRERO, A. y FEITO, R. (1996). La reforma y la formación permanente del profesorado. Revista de Educación, 309, pp. 29-37.
JIMÉNEZ, M.P. (1995). Comparando teorías: la reflexión sobre la naturaleza de la ciencia en la formación del profesorado, en Blanco, L. y Mellado, V.(eds.). Laformación del profesorado de ciencias y matemáticas en España y Portugal. Badajoz: Diputación Provincial.

KELLEY, H.H. (1972). Attribution in Social Interaction, en Jones, E., Kanouse, D., Kelley, H.H., Nisbett, R., Valins, S. y Weiner, B. (eds.). Attribution: Perceiving the Causes of Behaviour. Morristown, Nueva Jersey General Learning Press.

MARTÍNEZ, M.M., FERNÁNDEZ, M.P., GUERRERO, A., MARTÍN, R., RODRIGO, M. y VARELA, M.P. (1997). Estudio de la incidencia de distintos programas de formación y de la práctica educativa en el pensamiento del profesor de ciencias de educación secundaria. Memoria de investigación inédita. Madrid: CIDE.

MARTÍNEZ, M.M., FERNÁNDEZ, M.P., GUERRERO, A., MARTÍN, R., RODRIGO, M. y VARELA, M.P. (2001). ¿Qué pensamiento profesional y curricular tienen los futuros profesores de ciencias de secundaria? Enseñanza de las Ciencias, 19(1), pp. 67-87.

MEC (1992). Curso de actualización científica y didáctica. Ciencias de la naturaleza. (Carpeta de varias monografías). Madrid: MEC.

MELLADO, V. (1998). Preservice teachers'classroom practice and their conceptions of the nature of science, en Fraser, B.J. y Tobin, K.G. (ed.). International Handbook of Science Education. Gran Bretaña: Kluwer Academic Publishers, pp. 745-758.

MELLADO, V., RUIZ, C. y BLANCO, L.J. (1997). Aprender a enseñar ciencias experimentales en la formación inicial de maestros. Bordón, 49(3), pp. 275-288.

PÉREZ GÓMEZ, A.I. y GIMENO, J. (1992). El pensamiento pedagógico de los profesores: un estudio empírico sobre la incidencia de los cursos de aptitud pedagógica (CAP) y de la experiencia profesional en el pensamiento de los profesores. Investigación en la Escuela, 17, pp. 51-73.

PORLÁN, R., RIVERO, A. y MARTÍN DEL POZO, R. (1998). Conocimiento profesional y epistemología de los profesores II: Estudios empíricos y conclusiones. Enseñanza de las Ciencias, 16(2), pp. 271-288.

RODRIGO, M., MARTÍN, R., MARTÍNEZ, M.M., FERNÁNDEZ, M.P., GUERRERO, A. y VARELA, M.P. (2000). Un estudio sobre el profesor de ciencias de educación secundaria y unas propuestas para mejorar su formación. Revista de Educación, 321, pp. 291-314.

[Artículo recibido en diciembre de 2000 y aceptado en octubre de 2001.] 


\begin{tabular}{|c|c|c|c|c|c|}
\hline \multicolumn{6}{|c|}{$\begin{array}{c}\text { ANEXO } \\
\text { Cuestionario sobre la acción educativa* }\end{array}$} \\
\hline & SIEMPRE & FRECUENTEMENTE & A VECES & CASI NUNCA & NUNCA \\
\hline $\begin{array}{l}\text { 1. La evaluación de los alumnos la } \\
\text { realizo, fundamentalmente, a partir de } \\
\text { exámenes escritos }\end{array}$ & & & & & \\
\hline $\begin{array}{l}\text { 2. Utilizo algún instrumento específico } \\
\text { a la hora de evaluar las actitudes de los } \\
\text { alumnos relativas a las ciencias }\end{array}$ & & & & & \\
\hline $\begin{array}{l}\text { 3. La calificación del trabajo de } \\
\text { laboratorio lo utilizo como una parte de } \\
\text { la evaluación }\end{array}$ & & & & & \\
\hline $\begin{array}{l}\text { 4. En cada evaluación pido el cuaderno } \\
\text { de trabajo individual del alumno para } \\
\text { utilizarlo en la calificación }\end{array}$ & & & & & \\
\hline $\begin{array}{l}\text { 5. Las pruebas de evaluación las preparo } \\
\text { siguiendo mis propios criterios }\end{array}$ & & & & & \\
\hline $\begin{array}{l}\text { 6. Después de cada evaluación, analizo } \\
\text { los resultados con los compañeros de } \\
\text { seminario }\end{array}$ & & & & & \\
\hline $\begin{array}{l}\text { 7. Al final de cada evaluación, reviso } \\
\text { con los profesores del mismo nivel la } \\
\text { programación }\end{array}$ & & & & & \\
\hline $\begin{array}{l}\text { 8. La evaluación la realizo sólo para } \\
\text { comprobar si los alumnos han alcanzado } \\
\text { el nivel de conocimientos previstos }\end{array}$ & & & & & \\
\hline $\begin{array}{l}\text { 9. Después de cada evaluación, doy } \\
\text { opción a que los alumnos comenten } \\
\text { sobre los resultados de la misma }\end{array}$ & & & & & \\
\hline $\begin{array}{l}\text { 10. Uso los resultados de las } \\
\text { evaluaciones para informar a los } \\
\text { alumnos, individualmente, acerca de } \\
\text { sus dificultades }\end{array}$ & & & & & \\
\hline $\begin{array}{l}\text { 11. En los exámenes utilizo preguntas } \\
\text { encaminadas a evaluar contenidos sobre } \\
\text { procedimientos }\end{array}$ & & & & & \\
\hline $\begin{array}{l}\text { 12. Explico verbalmente cada tema } \\
\text { siguiendo un libro de texto o apuntes }\end{array}$ & & & & & \\
\hline $\begin{array}{l}\text { 13. Dada la distribución de tiempos y } \\
\text { alumnos generalmente trabajamos todos } \\
\text { en clase lo mismo y a la vez }\end{array}$ & & & & & \\
\hline $\begin{array}{l}\text { 14. Los alumnos realizan pequeñas } \\
\text { investigaciones en cada tema o unidad }\end{array}$ & & & & & \\
\hline $\begin{array}{l}\text { 15. Empleo problemas cotidianos como } \\
\text { recurso para enseñar ciencias }\end{array}$ & & & & & \\
\hline $\begin{array}{l}\text { 16. Al iniciar un tema, indago acerca de } \\
\text { las ideas de los alumnos para organizar } \\
\text { la enseñanza }\end{array}$ & & & & & \\
\hline $\begin{array}{l}\text { 17. Planifico mi enseñanza a partir de } \\
\text { lecciones }\end{array}$ & & & & & \\
\hline $\begin{array}{l}\text { 18. En el aula, desarrollo actividades } \\
\text { encaminadas a comprobar la } \\
\text { reestructuración de las ideas iniciales } \\
\text { de los alumnos }\end{array}$ & & & & & \\
\hline $\begin{array}{l}\text { 19. Los aspectos históricos de la ciencia } \\
\text { los utilizo solamente como un elemento } \\
\text { motivador }\end{array}$ & & & & & \\
\hline $\begin{array}{l}\text { 20. Organizo actividades que ilustren } \\
\text { sobre las implicaciones sociales de las } \\
\text { ciencias }\end{array}$ & & & & & \\
\hline
\end{tabular}




\begin{tabular}{|c|c|c|c|c|c|}
\hline & SIEMPRE & FRECUENTEMENTE & A VECES & CASI NUNCA & NUNCA \\
\hline $\begin{array}{l}\text { 21. Diseño actividades en las que los } \\
\text { alumnos tienen que aplicar lo aprendido } \\
\text { en el aula a problemas cotidianos }\end{array}$ & & & & & \\
\hline $\begin{array}{l}\text { 22. Las actividades prácticas las planteo } \\
\text { como comprobación posterior de los } \\
\text { aspectos explicados teóricamente }\end{array}$ & & & & & \\
\hline $\begin{array}{l}\text { 23. Los alumnos realizan las actividades } \\
\text { del aula de manera individual }\end{array}$ & & & & & \\
\hline $\begin{array}{l}\text { 24. Dedico atención específica a los } \\
\text { alumnos que presentan mayores } \\
\text { dificultades de aprendizaje }\end{array}$ & & & & & \\
\hline $\begin{array}{l}\text { 25. Propongo tareas diferentes a los } \\
\text { alumnos en función de sus características }\end{array}$ & & & & & \\
\hline $\begin{array}{l}\text { 26. Dada la distribución de tiempos y } \\
\text { alumnos, generalmente trabajamos todos } \\
\text { en clase lo mismo y a la vez }\end{array}$ & & & & & \\
\hline $\begin{array}{l}\text { 27. Permito que los estudiantes participen } \\
\text { y tomen decisiones sobre algunos } \\
\text { aspectos relativos a la marcha de la clase }\end{array}$ & & & & & \\
\hline $\begin{array}{l}\text { 28. Pongo a disposición de los } \\
\text { compañeros mis recursos y experiencias } \\
\text { personales }\end{array}$ & & & & & \\
\hline $\begin{array}{l}\text { 29. Las actividades para el aula las } \\
\text { organizo en: actividades de iniciación, } \\
\text { de desarrollo, de reestructuración y de } \\
\text { aplicación }\end{array}$ & & & & & \\
\hline $\begin{array}{l}\text { 30. En la puesta en práctica de mi plan } \\
\text { de trabajo utilizo los medios audiovisuales }\end{array}$ & & & & & \\
\hline $\begin{array}{l}\text { 31. En mi actividad docente empleo como } \\
\text { fuentes de información la prensa escrita } \\
\text { y revistas especializadas }\end{array}$ & & & & & \\
\hline $\begin{array}{l}\text { 32. Las salidas fuera del centro están } \\
\text { perfectamente integradas en mi } \\
\text { programación anual }\end{array}$ & & & & & \\
\hline $\begin{array}{l}\text { 33. En el desarrollo de las unidades } \\
\text { didácticas utilizo los medios informáticos }\end{array}$ & & & & & \\
\hline $\begin{array}{l}\text { 34. Los contenidos que explico a los } \\
\text { alumnos son una versión simplificada de } \\
\text { los conceptos más importantes de la } \\
\text { disciplina }\end{array}$ & & & & & \\
\hline $\begin{array}{l}\text { 35. Los contenidos que trabajo con los } \\
\text { alumnos son conceptos, procedimientos } \\
\text { y actitudes relevantes para la vida } \\
\text { cotidiana y la integración social de las } \\
\text { personas }\end{array}$ & & & & & \\
\hline $\begin{array}{l}\text { 36. En mis clases explico una versión } \\
\text { actualizada del conocimiento científico, } \\
\text { ya que es el conocimiento objetivo y } \\
\text { correcto }\end{array}$ & & & & & \\
\hline $\begin{array}{l}\text { 37. En mis clases introduzco cuestiones } \\
\text { históricas para poner de manifiesto el } \\
\text { carácter relativo y evolutivo del } \\
\text { conocimiento científico }\end{array}$ & & & & & \\
\hline $\begin{array}{l}\text { 38. Las ideas que tienen los alumnos } \\
\text { sobre los conceptos de ciencias son } \\
\text { errores que trato de sustituir por las ideas } \\
\text { correctas }\end{array}$ & & & & & \\
\hline $\begin{array}{l}\text { 39. Las ideas que manifiestan los alumnos } \\
\text { sobre los conceptos de ciencias son un } \\
\text { conocimiento alternativo al conocimiento } \\
\text { científico y al escolar }\end{array}$ & & & & & \\
\hline
\end{tabular}




\section{INVESTIGACIÓN DIDÁCTICA}

\begin{tabular}{|c|c|c|c|c|c|}
\hline & SIEMPRE & FRECUENTEMENTE & A VECES & CASI NUNCA & NUNCA \\
\hline $\begin{array}{l}\text { 40. La fuente de información que utilizo } \\
\text { para seleccionar los contenidos es el libro } \\
\text { de texto de igual o mayor nivel }\end{array}$ & & & & & \\
\hline $\begin{array}{l}\text { 41. Para seleccionar los contenidos utilizo } \\
\text { informaciones provenientes de los } \\
\text { estudios de las ideas de los alumnos, de } \\
\text { la historia de la ciencia o de otros } \\
\text { materiales curriculares que tienen en } \\
\text { cuenta aspectos de este tipo }\end{array}$ & & & & & \\
\hline $\begin{array}{l}\text { 42. Organizo los contenidos en una } \\
\text { secuencia que se ajusta a la lógica de la } \\
\text { disciplina }\end{array}$ & & & & & \\
\hline $\begin{array}{l}\text { 43. Organizo los contenidos en forma de } \\
\text { mapas que relacionan unos conceptos } \\
\text { con otros de acuerdo con el pensamiento } \\
\text { de los alumnos }\end{array}$ & & & & & \\
\hline $\begin{array}{l}\text { 44. El nivel de conocimientos al que } \\
\text { tienen que llegar los alumnos es el } \\
\text { prefijado en la programación }\end{array}$ & & & & & \\
\hline $\begin{array}{l}\text { 45. Evalúo positivamente a los alumnos } \\
\text { cuando experimentan una evolución } \\
\text { favorable de sus propias ideas, aunque } \\
\text { no hayan alcanzado el nivel esperado }\end{array}$ & & & & & \\
\hline $\begin{array}{l}\text { 48. La formación científica recibida en } \\
\text { la universidad es suficiente para poder } \\
\text { desempeñar la labor docente en la } \\
\text { enseñanza secundaria }\end{array}$ & & & & & \\
\hline $\begin{array}{l}\text { 49. El profesorado de enseñanza } \\
\text { secundaria necesita una formación psico- } \\
\text { pedagógica suplementaria a su formación } \\
\text { científica para desempeñar su función }\end{array}$ & & & & & \\
\hline $\begin{array}{l}\text { 50. La procedencia socioeconómica de } \\
\text { los alumnos es la principal razón de su } \\
\text { rendimiento escolar }\end{array}$ & & & & & \\
\hline $\begin{array}{l}\text { 51. Aunque chicas y chicos parecen tener } \\
\text { la misma capacidad para el aprendizaje } \\
\text { de las ciencias, las chicas muestran una } \\
\text { actitud más favorable hacia las mismas }\end{array}$ & & & & & \\
\hline $\begin{array}{l}\text { 52. La mayor inclinación que presentan } \\
\text { los chicos respecto a las chicas en las } \\
\text { disciplinas de ciencias refleja sus mejores } \\
\text { aptitudes en esta área }\end{array}$ & & & & & \\
\hline $\begin{array}{l}\text { 53. En el área de ciencias es más } \\
\text { importante poseer una buena aptitud que } \\
\text { en otras áreas curriculares }\end{array}$ & & & & & \\
\hline $\begin{array}{l}\text { 55. El éxito o fracaso de los alumnos } \\
\text { depende básicamente de sus } \\
\text { características personales (inteligencia, } \\
\text { motivación...) }\end{array}$ & & & & & \\
\hline $\begin{array}{l}\text { 56. Un profesor de ciencias dispone de } \\
\text { recursos suficientes para hacer rendir } \\
\text { adecuadamente a los alumnos, con } \\
\text { independencia de la extracción social o } \\
\text { características personales de éstos }\end{array}$ & & & & & \\
\hline $\begin{array}{l}\text { 57. El mayor fracaso de los alumnos en } \\
\text { las asignaturas de ciencias se debe } \\
\text { fundamentalmente a que en su enseñanza } \\
\text { se utilizan métodos inadecuados }\end{array}$ & & & & & \\
\hline $\begin{array}{l}\text { 60. La personalidad y las actitudes del } \\
\text { profesor de ciencias tiene menor } \\
\text { incidencia en el rendimiento de los } \\
\text { alumnos que sus conocimientos } \\
\text { científicos y didácticos }\end{array}$ & & & & & \\
\hline
\end{tabular}




\begin{tabular}{|c|c|c|c|c|c|}
\hline & SIEMPRE & FRECUENTEMENTE & A VECES & CASI NUNCA & NUNCA \\
\hline $\begin{array}{l}\text { 61. Las dificultades de las materias de } \\
\text { ciencias se deben en gran medida al } \\
\text { lenguaje técnico propio de estas } \\
\text { disciplinas }\end{array}$ & & & & & \\
\hline $\begin{array}{l}\text { 62. El desarrollo cognitivo de los alumnos } \\
\text { es el principal responsable de su } \\
\text { rendimiento }\end{array}$ & & & & & \\
\hline $\begin{array}{l}\text { 63. Una de las finalidades importantes de } \\
\text { las asignaturas de ciencias es potenciar } \\
\text { el pensamiento crítico de los alumnos }\end{array}$ & & & & & \\
\hline $\begin{array}{l}\text { 64. Del conjunto de las áreas curriculares, } \\
\text { las asignaturas de ciencias son las que } \\
\text { contribuyen, en mayor medida, al ejercicio } \\
\text { de la mente }\end{array}$ & & & & & \\
\hline
\end{tabular}

\section{* Notas}

- Las opciones seleccionadas y sombreadas corresponden a la denominada tendencia constructivista.

- Las preguntas que aquí aparecen ordenadas, para su mejor consulta, en las encuestas estaban distribuidas al azar y se corresponden con: ítems del 1 al 11 (dimensión de evaluación); ítems del 12 al 33 (dimensión de metodología); ítems del 34 al 45 (dimensión de contenidos); e ítems del 48 al 64 , (percepciones profesionales). 